\title{
EFFICIENT DECOUPLING OF MULTIPHYSICS SYSTEMS FOR UNCERTAINTY PROPAGATION
}

\author{
A Thesis \\ by \\ SAMUEL ISAAC FRIEDMAN \\ Submitted to the Office of Graduate and Professional Studies of \\ Texas A\&M University \\ in partial fulfillment of the requirements for the degree of \\ MASTER OF SCIENCE
}

Chair of Committee, Douglas Allaire

Committee Members, Raymundo Arroyave Astrid Layton

Head of Department, Andreas Polycarpou

May 2019

Major Subject: Mechanical Engineering

Copyright 2019 Samuel Isaac Friedman 


\begin{abstract}
Uncertainty propagation through coupled multiphysics systems is often intractable due to computational expense. In this work, we present a novel methodology to enable uncertainty analysis of expensive coupled systems. The approach consists of offline discipline level analyses followed by an online synthesis that results in accurate approximations of full coupled system level uncertainty analyses. Coupling is handled by an efficient procedure for approximating the map from system inputs to fixed point sets that makes use of state of the art $\ell_{1}$-minimization techniques and cut high dimensional model representations. The methodology is demonstrated on an analytic numerical example and a fire detection satellite system where it is shown to perform well as compared to brute force Monte Carlo simulation.
\end{abstract}




\section{ACKNOWLEDGMENTS}

I would like to thank my advisor and committee chair, Dr. Douglas Allaire, for his invaluable support and guidance throughout my time at Texas A\&M. I would also like to thank my committee

members Dr. Raymundo Arroyave and Dr. Astrid Layton for their support and encouragement in this process.

I would like to thank Dr. Daniel Hogenauer for helping me begin my engineering career.

I would like to thank my siblings, Asher, Sydney, and Rachel and my parents, Dr. David Friedman and Elizabeth Stool Friedman, for their generous and loving support throughout my entire academic journey.

Finally, I would like to thank my partner, Leanna Garb, for her unwavering support, encouragement, kindness, and love. 


\section{CONTRIBUTORS AND FUNDING SOURCES}

\section{Contributors}

This work was supported by a thesis committee consisting of Professor Douglas Allaire and Professor Astrid Layton of the Department of Mechanical Engineering and Professor Raymundo Arroyave of the Department of Materials Science \& Engineering.

All other work conducted for the thesis was completed by the student independently.

\section{Funding Sources}

Graduate study was supported by the Air Force Office of Scientific Research MURI on multiinformation sources of multi-physics systems under Award Number FA9550-15-1-0038, program manager Dr. Fariba Fahroo. 


\section{TABLE OF CONTENTS}

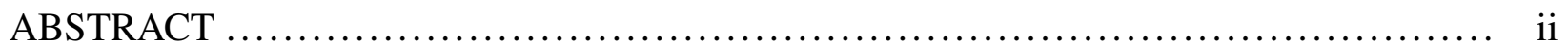

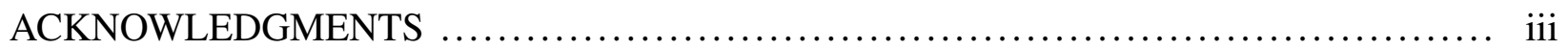

CONTRIBUTORS AND FUNDING SOURCES $\ldots \ldots \ldots \ldots \ldots \ldots \ldots \ldots \ldots \ldots \ldots \ldots \ldots \ldots \ldots \ldots \ldots$

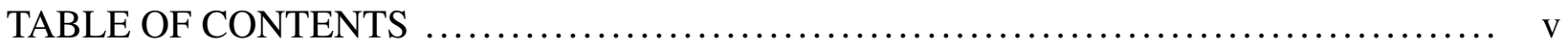

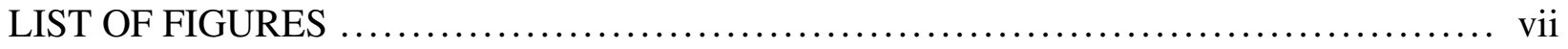

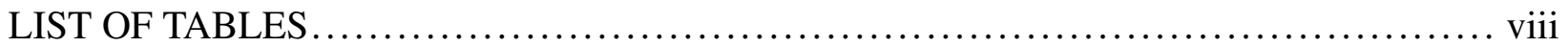

1. INTRODUCTION AND LITERATURE REVIEW $\ldots \ldots \ldots \ldots \ldots \ldots \ldots \ldots \ldots \ldots \ldots \ldots \ldots$

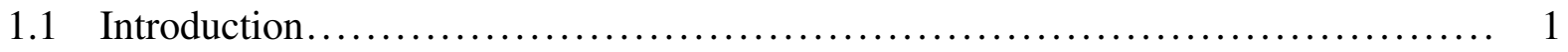

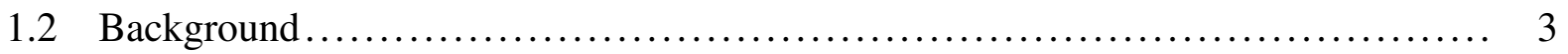

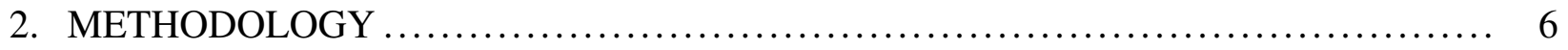

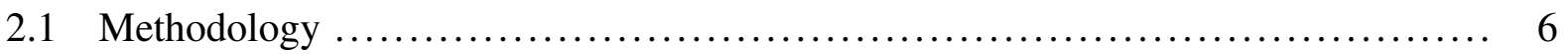

2.1.A Approximation of Coupling Variable Fixed Point Sets for Decoupling Mul-

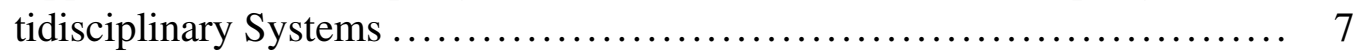

2.1.A.1 Cut High Dimensional Model Representations ................. 7

2.1.A.2 Sparse Representation of Each Subfunction via $\ell_{1}$-Minimization .. 8

2.1.B Kernel Density Estimation for Density Ratio Approximation.............. 10

2.1.C Estimate Statistics For Quantities of Interest and Find Joint Densities ....... 11

2.1.D Algorithm ................................................. 13

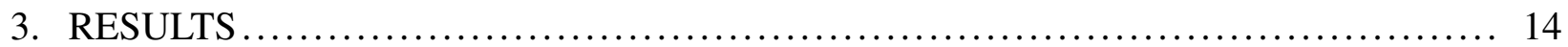

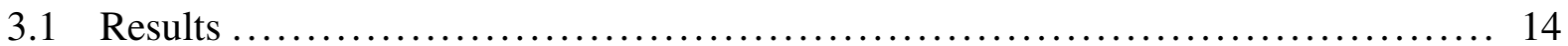

3.1.A Analytical Example .......................................... 14

3.1.B Fire Detection Satellite Example ............................... 16

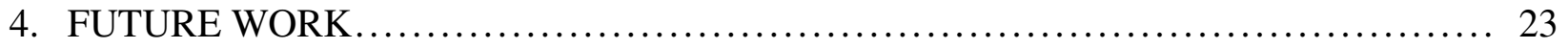

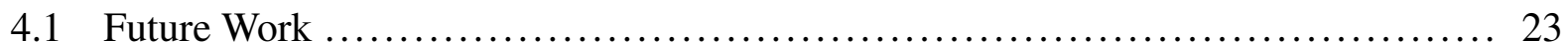

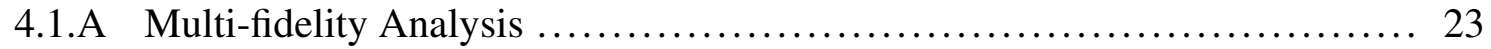

4.1.B Network Topology Analysis .................................. 23

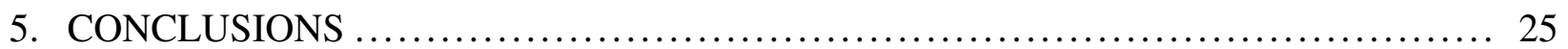




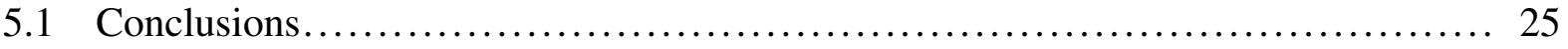

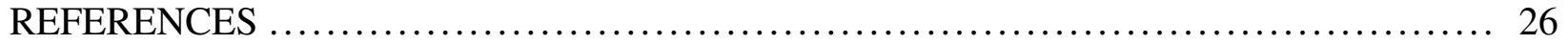




\section{LIST OF FIGURES}

FIGURE

1.1 System of coupled disciplines. On the left is a fully coupled system and on the right is a decomposed, or decoupled system that does not require any iterative resolution

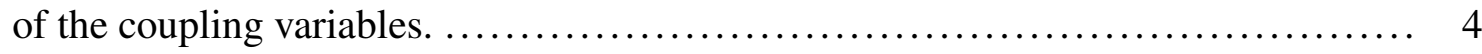

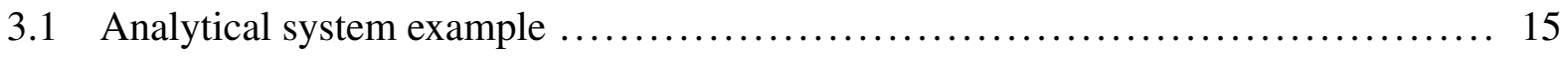

3.2 Cumulative distribution function of coupling variables $y_{12}$ and $y_{21}$, and quantities of interest $q_{1}$ and $q_{2}$ of analytical system example........................ 16

3.3 Fire detection satellite model $\ldots \ldots \ldots \ldots \ldots \ldots \ldots \ldots \ldots \ldots \ldots \ldots \ldots \ldots \ldots \ldots \ldots \ldots$

3.4 Fire Satellite: cumulative distribution functions of coupling variables: power other than attitude control, $P_{\mathrm{ACS}}$, maximum moment of inertia, $I_{\max }$ and minimum mo-

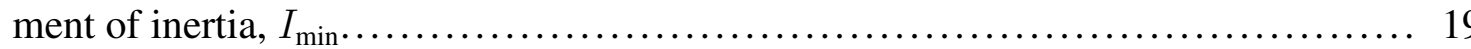

3.5 Fire Satellite: cumulative distribution functions of quantities of interest: total

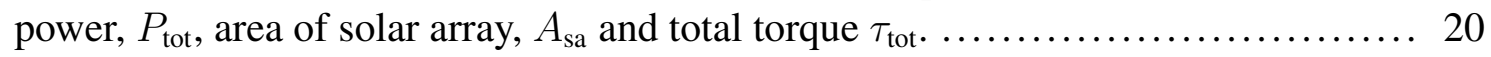

3.6 Illustration of importance weighting offline samples for the joint density of fire satellite model quantities of interest: area of the solar array, $A_{\mathrm{sa}}$, and total torque, $\tau_{\text {tot }} .22$

4.1 High-fidelity drop-in discipline replacement for fire satellite problem ............ 24 


\section{LIST OF TABLES}

TABLE

Page

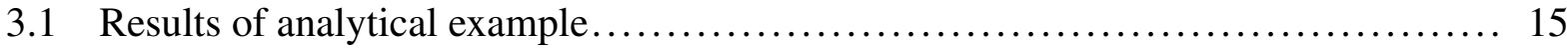

3.2 Fire detection satellite input variables as modified from Ref. [1] $\ldots \ldots \ldots \ldots \ldots \ldots \ldots, 18$

3.3 Results of fire satellite example, coupling variables $\ldots \ldots \ldots \ldots \ldots \ldots \ldots \ldots \ldots \ldots \ldots$

3.4 Results of fire satellite example, quantities of interest $\ldots \ldots \ldots \ldots \ldots \ldots \ldots \ldots \ldots \ldots$ 


\section{INTRODUCTION AND LITERATURE REVIEW*}

\subsection{Introduction}

In an effort to create higher performing aerospace systems, many new technologies seek to exploit interactions among coupled disciplines. Often, there is great uncertainty associated with the analysis of such new capabilities, which must be taken into account. To properly quantify this uncertainty, high fidelity computational tools are essential given that the design emphasis is on the identification of synergistic emerging phenomena that likely cannot be captured adequately by low fidelity tools. For systems with feedback coupling, uncertainty propagation with expensive computational models can be computationally prohibitive. Thus, there is a critical need for the development of efficient methods for incorporating high fidelity information in the uncertainty analysis of feedback coupled multiphysics systems.

The uncertainty analysis for coupled multiphysics systems requires the propagation of uncertainty from model inputs to model outputs. There are many sources of uncertainty that should be considered for such an analysis. For computational models, these include, parametric uncertainty, parametric variability, code uncertainty, and model discrepancy [3]. Here, we focus on parametric uncertainty and note that much of what we propose can be extended to handle the other forms of uncertainty. Often, multiphysics simulations are composed of initially independent disciplinary computational models. These models are coupled via some set of variables that are involved in calculations required for more than one disciplinary analysis. For such systems, the task of uncertainty analysis can be challenging for many reasons. For example, disciplinary models may be housed in different locations, analysis capabilities may run on different platforms and have significantly different runtimes, and the sheer number of disciplines can make the process difficult to manage. Uncertainty analysis for such coupled systems usually begins by composing the disciplinary models and creating a capability to perform techniques such as fixed point iteration to ensure compatibility of shared coupling variables. This step is burdensome from the perspec-

\footnotetext{
${ }^{*}$ Reprinted from Ref. [2]
} 
tive of managing the entire system and also from the significant computational expense that arises from such iterative approaches. For uncertainty analysis, the propagation of thousands of samples through a fixed point iteration process is almost certainly computationally prohibitive.

To mitigate these issues in the uncertainty analysis of expensive to evaluate coupled systems, we propose an efficient decoupling of multiphysics systems followed by a compositional uncertainty analysis that constructs results from discipline level model evaluations as if they were drawn from the full coupled system. The work builds off of Refs. $[4,5,6,7,8]$, where decompositionbased methodologies are presented for both feed-forward and feedback coupled systems. The work presented here is a natural extension of Ref. [5] for the case of coupled systems under parametric uncertainty.

The key features of our work include an offline/online approach to uncertainty analysis of coupled systems that results in significant computational savings. Further, the methodology presented here enables the rapid and accurate replacement of a given disciplinary model with another, perhaps higher fidelity or more trusted disciplinary model. This is an important concept in multifidelity methods, were many models can potentially be used. A full exploration of this alternate model drop-in capability is a topic for future work and discussed in Section 4.1. Here, our approach builds from offline uncertainty analyses of each individual discipline. In an online phase, the fixed point sets of the coupled system are identified in a goal-oriented manner following the approach of [9]. This approach uses $\ell_{1}$-minimization and cut high dimensional model representations to ensure as few samples as possible are used in finding the fixed point sets. Once this is accomplished, the map from inputs to fixed point sets is used to quantify online the correct joint densities that should have been used in the offline discipline level uncertainty analyses. Importance reweighting is then used via the Radon-Nikodym derivative (density ratio) of the offline and online densities. The result is a full coupled system uncertainty analysis at the expense of only a few online model evaluations. We demonstrate the full methodology on a known analytical example problem and on a multidisciplinary fire detection satellite system.

The rest of the paper is organized as follows. Section 1.2 presents background on related work 
as well as a discussion of the general problem setup. The methodology is presented in Section 2.1. In Section 3.1 we present the demonstrations, and in Section 4.1 we discuss opportunities for future work. Conclusions are drawn in Section 5.1.

\subsection{Background}

Previous work on multidisciplinary uncertainty analysis has focused on approximations such as surrogate modeling and simplified representations of system uncertainty. The use of surrogates for disciplinary models in a composed system can provide computational savings, as well as simplify the task of integrating components [10]. Approximate representations of uncertainty, such as using mean and variance information in place of a full probability distribution have been used to avoid the need to propagate uncertainty between disciplines. Such simplifications are commonly used in uncertainty-based multidisciplinary design optimization methods as a way to avoid a systemlevel uncertainty analysis [11]. These approaches include implicit uncertainty propagation [12], reliability-based design optimization [13], robust moment matching [14, 15, 16], advanced mean value method [17], collaborative reliability analysis using most probable point estimation [18], and a multidisciplinary first-order reliability method [19].

Other recent work has focused on exploiting the structure of a given multidisciplinary system. Ref. [1] presents a likelihood-based approach to decouple feedback loops, thus reducing the problem to a feed-forward system. Dimension reduction and measure transformation to reduce the dimensionality and propagate the coupling variables between coupled components have been performed in a coupled feedback problem with polynomial chaos expansions [20, 21, 22]. Coupling disciplinary models by representing coupling variables with truncated Karhunen-Loève expansions, has been studied for multiphysics systems [23]. A hybrid method that combines Monte Carlo sampling and spectral methods for solving stochastic coupled problems has also been proposed by Refs. [24] and [25].

Our approach builds on the work of Refs. $[4,5,6]$, where the challenges of uncertainty analysis for feed-forward multidisciplinary systems were dealt with using a decomposition-based approach. Without loss of generality, the emphasis in this paper is on a system of two coupled disciplines. 
Extensions to systems with more complex architectures do not require any modifications to our methodology, but will incur more computational expense due to the need to resolve more coupling variables. In the case considered here, each discipline takes in a system-level input and a feedback coupled variable. Such a system is shown notionally in Fig. 1.1a, where two disciplines share two variables. The discipline outputs are a system-level quantity of interest and a dependent variable for the other discipline in the system. Parametric uncertainty is associated with the input vector to the system. A standard method for finding the joint distribution of the quantities of interest under uncertainty would be to implement a nonlinear equation solver, such as fixed point iteration, for a distribution of uncertain inputs $\mathbf{x}=\left(x_{1}, x_{2}, \ldots, x_{d}\right)$. This direct approach requires several individual discipline evaluations for each input sample, $\mathbf{x}_{i}$, several thousand of which may be required for accurate statistical analysis. Expensive, high-fidelity disciplines can quickly render this approach intractable for large sample sizes. Solving the system of variables in its coupled state, as in Fig. 1.1a, while costly, incorporates the dependent nature of the discipline inputs and outputs in the resulting joint density of the quantities of interest.

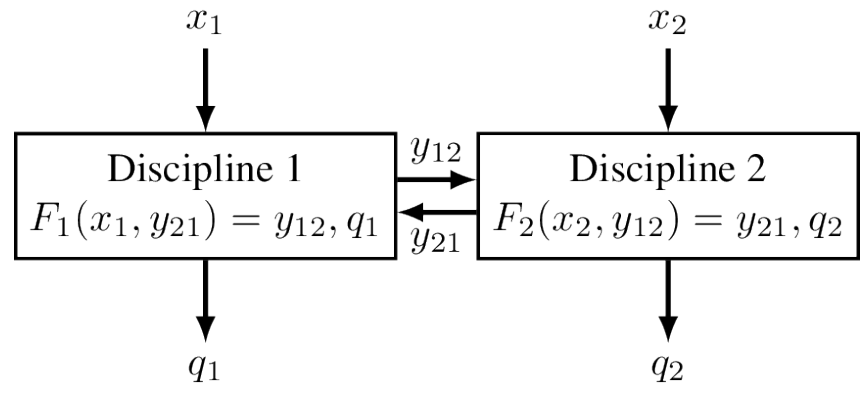

(a) Coupled State
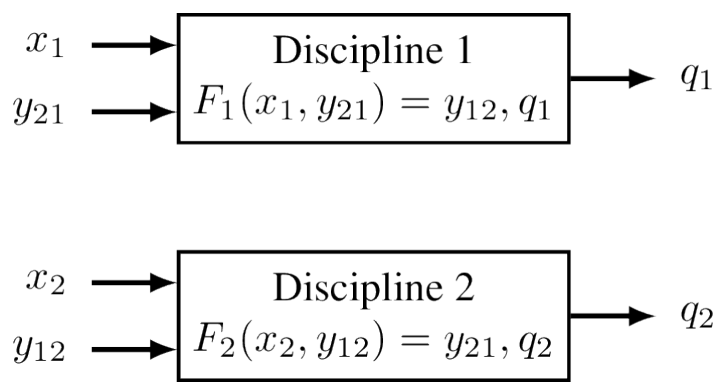

(b) Decoupled State

Figure 1.1: System of coupled disciplines. On the left is a fully coupled system and on the right is a decomposed, or decoupled system that does not require any iterative resolution of the coupling variables.

Evaluating each discipline independently in a decoupled state, as in Fig. 1.1b, will produce independent distributions of the quantities of interest. This result is practical to achieve, but does 
not reflect the reality of the inherently coupled multiphysics system and will produce inaccurate results. However, if there exist a bank of previously evaluated "offline" samples for each decoupled discipline, we can assign weights to those samples to reflect the dependence in the full coupled system. Our goal is to find these importance weights that reflect the dependence in the system with minimal full system-level evaluations. We will compare the accuracy of the quantities of interest when the method is applied to that of brute force Monte Carlo simulation. 


\section{METHODOLOGY*}

\subsection{Methodology}

The goal of our method is to take advantage of existing samples from previous evaluations of each discipline in a decoupled state. Examining each discipline individually, the inputs can be either system-level stochastic variables or intermediate variables that are the output of another discipline in the system. The outputs of the decoupled discipline are assumed to be a system-level quantity of interest. The intermediate coupling variables are considered independent when they are inputs to disciplines in a decoupled state, although they are dependent on other disciplines when considered as part of the larger system of disciplines. The quantities of interest found at the discipline level could have different probability densities from those found at the system level, which account for dependent interactions between disciplines. Our method accounts for dependent system-level interactions between disciplines in the system by assigning weights to existing discipline-level evaluations. The result is an accurate approximation of system-level quantities of interest without new model evaluations. If our system features feedback coupling between disciplines, then a full system evaluation may involve many more individual discipline evaluations than would be required in an equivalent decoupled setup. Thus, to enable taking advantage of previously computed discipline level samples, our objective is also to find accurate approximations of the fixed point sets of the coupling variables with minimal full system evaluations, thereby reducing the required online computational expense.

In the following subsections, the ingredients of our overall approach are presented. These ingredients include cut high dimensional model representations, compressed sensing via $\ell_{1}$ minimization, kernel density estimation, and importance reweighting. We conclude this section with a high level algorithm that outlines the steps of our process.

${ }^{*}$ Reprinted from Ref. [2] 


\subsection{A Approximation of Coupling Variable Fixed Point Sets for Decoupling Multidisci- plinary Systems}

Our goal here is to identify the map from system inputs to resolved coupling variables. We seek to do this with as few samples (each of which requires a fixed point iteration) as possible. Following [9], we achieve this by building cut high dimensional model representations with only univariate and bivariate terms. To build these terms, we use $\ell_{1}$-minimization with a controllable error tolerance. Each technique is described below.

\subsection{A.1 Cut High Dimensional Model Representations}

The most general high dimensional model representation (HDMR) is given as $[26,27,28]$

$$
\begin{aligned}
f\left(x_{1}, x_{2}, \ldots, x_{d}\right) & =f_{0}+\sum_{j=1}^{d} f_{j}\left(x_{j}\right)+\sum_{j<l}^{d} f_{j, l}\left(x_{j}, x_{l}\right)+\cdots+f_{1,2, \ldots, d}\left(x_{1}, x_{2}, \ldots, x_{d}\right) \\
& =\sum_{\mathbf{u} \subseteq \mathcal{D}} f_{\mathbf{u}}\left(\mathbf{x}_{\mathbf{u}}\right),
\end{aligned}
$$

where $\mathcal{D}:=\{1,2, \ldots, d\}$ denotes the set of input indices, $\mathbf{u}$ is a multi-index, and individual terms in each summand are referred to as subfunctions. Assuming that a map between inputs and fixed points in the coupling variable space has low superposition dimension simplifies the problem into an effective superposition dimension [29]. This dimension is defined as the smallest integer, $d_{s}$, such that some specified percentage of the variability of the function is captured by all terms in the HDMR with $d_{s}$ or less variables. Here, we assume $d_{s}=2$, and thus, consider only univariate and bivariate subspaces in the input space.

The specific form of the HDMR we use here is the cut-HDMR. This particular HDMR makes use of a Dirac measure, which establishes a "cut vector", also referred to as an "anchor point". If the cut vector, which is user defined and often a nominal point in the input space, is given as $\mathbf{x}^{c}$, and the value of the function at the cut vector is defined as $f_{0}=f\left(\mathbf{x}^{c}\right)$, then the $d_{s}=2$ cut-HDMR 
subfunctions can be written as

$$
\begin{aligned}
f_{j}\left(x_{j}\right) & =f\left(x_{j} ; \mathbf{x}^{c} \backslash x_{j}\right)-f_{0} \\
f_{j, l}\left(x_{j}, x_{l}\right) & =f\left(x_{j}, x_{l} ; \mathbf{x}^{c} \backslash x_{j}, x_{l}\right)-f_{i}-f_{j}-f_{0},
\end{aligned}
$$

for all $j, l \in \mathcal{D}$, where $\left(x_{j} ; \mathbf{x}^{c} \backslash x_{j}\right)$ refers to the cut vector with the $j^{\text {th }}$ entry free to vary. The approximation constructed by univariate and bivariate subfunctions can then be written as

$$
f\left(x_{1}, x_{2}, \ldots, x_{d}\right) \approx \hat{f}\left(x_{1}, x_{2}, \ldots, x_{d}\right)=f_{0}+\sum_{j=1}^{d} f_{j}\left(x_{j}\right)+\sum_{j<l}^{d} f_{j, l}\left(x_{j}, x_{l}\right)
$$

\subsection{A.2 Sparse Representation of Each Subfunction via $\ell_{1}$-Minimization}

The individual subfunctions may be calculated using fixed point iterations, which is a computationally expensive task. In our approach we achieve this by finding sparse representations to each subfunction in Eqs. 2.3 and 2.4. This is done by interrogating each subspace along the univariate directions from the cut vector and likewise with the bivariate terms. Thus, each term in Eq. (2.5) will have its own sparse representation.

Following Ref. [30], we provide a concise overview of the technique as applied to function approximation using compressed sensing. Let us represent individual subfunctions as $g$. The general concept is that the fixed point sets in coupled systems can be approximated well by a sparse representation in some functional basis. If this is the case, then the coefficient vector in the

functional basis requires only a few nonzero entries. For a given set of basis functions, $\left\{\psi_{k}\right\}_{k=1}^{N}$, we assume that the individual subfunctions, $g$, can be represented as a linear combination

$$
g=\Psi \mathbf{c}
$$

where $\Psi$ is an $N \times N$ matrix with columns, $\psi_{k}$, and $\mathbf{c}$ is an $N \times 1$ vector of coefficients. If $g$ is sparse in the basis, $\Psi$, then $\mathrm{c}$ will consist of many values that are effectively zero. The function 
is called $S$-sparse in $\Psi$ if there exists a c $\in \mathbb{R}^{N}$ with only $S<<N$ nonzero entries. Samples of the signal, $g$, are obtained by another linear operator, $\Phi$, which is an $M \times N$ measurement matrix, where $M<N$. A requirement of compressed sensing is that $\Phi$ and $\Psi$ be as incoherent as possible, which is accomplished in practice by randomly sampling the inputs of the system, which is equivalent to randomly sampling from a larger set of input-output samples. Then the sampled signal is

$$
\mathbf{b}=\Phi g
$$

which in our context, is just $M$ fixed point solutions of our computational model. That is, $M$ different input sets whose associated coupling variables are found via fixed point iteration. The purpose of compressed sensing is then to recover the sparsest signal, $\Psi \mathbf{c}$, that produces the measurements $g$. This can be written as an optimization problem as

$$
\hat{\mathbf{c}}=\arg \min _{\mathbf{c} \in \mathbb{R}^{N}}\|\mathbf{c}\|_{0} \text { subject to } \mathbf{b}=\Phi \Psi \mathbf{c},
$$

where $\|\mathbf{c}\|_{0}$ is defined as the number of nonzero entries in c. Finding a solution to this problem would require enumeration of all possibilities and is thus of combinatorial complexity. However, using compressed sensing, we can achieve this by the convex relaxation of Eq. 2.8 by using the $l_{1}$ norm to find the coefficients as

$$
\hat{\mathbf{c}}=\arg \min _{\mathbf{c} \in \mathbb{R}^{N}}\|\mathbf{c}\|_{1} \text { subject to } \mathbf{b}=\Phi \Psi \mathbf{c}
$$

where, $\|\mathbf{c}\|_{1}=\sum_{k=1}^{N}\left|\mathbf{c}_{k}\right|$. With enough measurements, if $g$ is sparse in $\Psi$, then it can nearly always be reconstructed from b using Eq. (2.9), as $g \approx \Psi \hat{\mathbf{c}}$ [31]. Equation (2.9) can be implemented as a linear program, for which many efficient solution algorithms exist.

If the function to be approximated is sparse in a given basis representation, then Eq. (2.9) can used to find the representation with remarkably few samples. However, if the appropriate basis representation is not known (or does not exist) the method will fail to find a useful approximation. 
Thus, we propose to use a more goal-oriented version of $\ell_{1}$-minimization from the compressed sensing field. Specifically, we consider the case where some error is permitted in the final approximation by instead solving

$$
\hat{\mathbf{c}}=\underset{c \in \mathbb{R}^{N}}{\arg \min }\|\mathbf{c}\|_{1} \quad \text { subject to } \quad\|\Phi \Psi \mathbf{c}-\mathbf{b}\|_{2} \leq \epsilon .
$$

Here, $\epsilon>0$ is a predetermined level of acceptable error in the approximation. By tailoring this error term to our specific problem we are eventually ensured of finding a sparse approximation to the fixed point sets without having to gather any more data from the full coupled system. We focus our efforts on univariate and bivariate subspaces and assume the underlying maps are approximately additive to ensure computational efficiency.

\subsection{B Kernel Density Estimation for Density Ratio Approximation}

A key feature of our work is the reweighting of offline samples of discipline level inputs and outputs. Prior to the identification of the coupled system fixed point sets, the joint distributions of these disciplines are unknown. Thus, independent joint densities, referred to as the proposal density, are used for uncertainty propagation through each discipline. To reweight these samples with the correct input distributions, or target distributions, we estimate the correct joint density using a general multivariate kernel density estimator trained with samples propagated through the learned map from inputs to resolved coupling variables discussed previously. Following Ref. [32], the general multivariate kernel density estimator we implement is given as

$$
\hat{f}(\mathbf{x})=\frac{1}{n|H|} \sum_{i=1}^{n} K\left(H^{-1}\left(\mathbf{x}-\mathbf{x}_{i}\right)\right)
$$

where $H$ is the bandwidth estimator and has the form of a $d \times d$ nonsingular matrix, $K$ is a kernel function, $K: \mathbb{R}^{d} \rightarrow \mathbb{R}^{1}$. Our multivariate implementation uses a product kernel, which is the product of one-dimensional kernels for each dimension: $K(x)=k\left(x_{1}\right) k\left(x_{2}\right) \cdots k\left(x_{d}\right)$. In this case, the bandwidth matrix $H$ is a diagonal matrix with the elements of each dimension's 
bandwidth on the diagonal.

For large samples, the choice of kernel has less impact on the accuracy of the estimate than the choice of bandwidth. We used a standard Gaussian kernel, $\mathcal{N}(0,1)$, for the multivariate density estimate. A topic of future work is to explore the use of different kernels for the density estimate, such as the Epanechnikov kernel, $\frac{3}{4}\left(1-t^{2}\right)$, which is more computationally efficient while sacrificing some accuracy and has only finite support [32]. For multivariate density estimates with multivariate normal kernels, the reference rule [32] for calculating bandwidth, $h_{j}$, for each input variable $j$ is

$$
h_{j}=\sigma_{j}\left(\frac{4}{n(d+2)}\right)^{1 /(d+4)}
$$

where $n$ is the number of samples, $d$ is the number of variables, and $\sigma_{j}$ is the standard deviation for variable $j$. We use the kernel density estimate to estimate the joint probability density values of the joint density derived from the cut-HDMR evaluated at the offline sample points. The result is a vector of joint density values that becomes the target distribution in the importance weighting step.

The importance weights for the offline samples are calculated by dividing the target density by the proposal density at each offline sample point. To calculate the statistics of the quantities of interest, the weights must be normalized such that $\sum_{i}^{n} w\left(\mathbf{x}_{i}\right)=1$. The weights are normalized by dividing each one by the sum of the weight vector as

$$
w\left(\mathbf{x}_{i}\right) \propto \frac{\pi\left(\mathbf{x}_{i}\right)}{p\left(\mathbf{x}_{i}\right)} \quad \Longrightarrow \quad w\left(\mathbf{x}_{i}\right)=\left[\sum_{i}^{n} \frac{\pi\left(\mathbf{x}_{i}\right)}{p\left(\mathbf{x}_{i}\right)}\right]^{-1} \frac{\pi\left(\mathbf{x}_{i}\right)}{p\left(\mathbf{x}_{i}\right)}
$$

where $\mathbf{x}_{i}$ is the vector of offline samples, $\pi(\cdot)$ is the target density from the kernel density estimate, and $p(\cdot)$ is the proposal density from the previously acquired offline sample data.

\subsection{Estimate Statistics For Quantities of Interest and Find Joint Densities}

Once we have the vector of normalized weights, $\mathbf{w}=\left[w\left(\mathbf{x}_{1}\right), w\left(\mathbf{x}_{2}\right), \ldots, w\left(\mathbf{x}_{n}\right)\right]^{\top}$, we can directly approximate the first and second moments of the quantities of interest $[33,34]$. The derivation of the equation to calculate the mean for the weighted quantity of interest begins with the 
expectation of the quantity of interest, $q(\mathbf{x})$, evaluated with respect to the target distribution $\pi(\mathbf{x})$ given as

$$
\mathbb{E}_{\pi}[q(\mathbf{x})]=\int_{\Pi} \pi(\mathbf{x}) q(\mathbf{x}) d \mathbf{x}=\int_{\mathcal{P}} \frac{\pi(\mathbf{x})}{p(\mathbf{x})} q(\mathbf{x}) p(\mathbf{x}) d \mathbf{x}
$$

where $\mathcal{P}$ is the support of the proposal density, $\Pi$ is the support of the target distribution, and the target distribution is absolutely continuous with respect to the proposal distribution. We can evaluate this integral with Monte Carlo simulation as

$$
\mathbb{E}_{\pi}[q(\mathbf{x})] \approx \frac{1}{n} \sum_{i=1}^{n} w\left(\mathbf{x}_{i}\right) q_{\mathrm{off}}\left(\mathbf{x}_{i}\right)
$$

where $q_{\text {off }}$ represents the offline samples of the given quantity of interest. Similarly, we can estimate the variance of the quantity of interest as

$$
\operatorname{Var}_{\pi}[q(\mathbf{x})]=\sum_{i}^{n}\left[w\left(\mathbf{x}_{i}\right)\left(q_{\text {off }}\left(\mathbf{x}_{i}\right)\right)^{2}\right]-\left[\sum_{i}^{n} w\left(\mathbf{x}_{i}\right) q_{\text {off }}\left(\mathbf{x}_{i}\right)\right]^{2} .
$$

The approximated distribution and density of the quantities of interest can be obtained via the weighted empirical distribution function. The weighted empirical cumulative distribution function $(\mathrm{CDF})$ is calculated from the offline quantity of interest data, $q_{\text {off }}$, and the weight vector, w, as

$$
F_{w}(\mathbf{t})=\frac{1}{n} \sum_{i=1}^{n} w_{i} \mathbb{I}\left(\mathbf{x}_{i} \leq \mathbf{t}\right)
$$

Here, $w_{i}$ is the importance weight for the $i^{\text {th }}$ sample, $n$ is the number of random samples, and $\mathbb{I}(\cdot)$ is the maximum convention heavyside step function defined as

$$
\mathbb{I}\left(\mathbf{x}_{i} \leq \mathbf{t}_{i}\right)= \begin{cases}1 & \text { if } x_{i} \leq t_{i}, \forall i \in 1,2, \ldots, d \\ 0 & \text { otherwise }\end{cases}
$$

Ref. [35] contains a proof that shows that as $n \rightarrow \infty$, the weighted empirical CDF of the proposal distribution converges to the desired target distribution. Once the CDF is obtained, it can be 


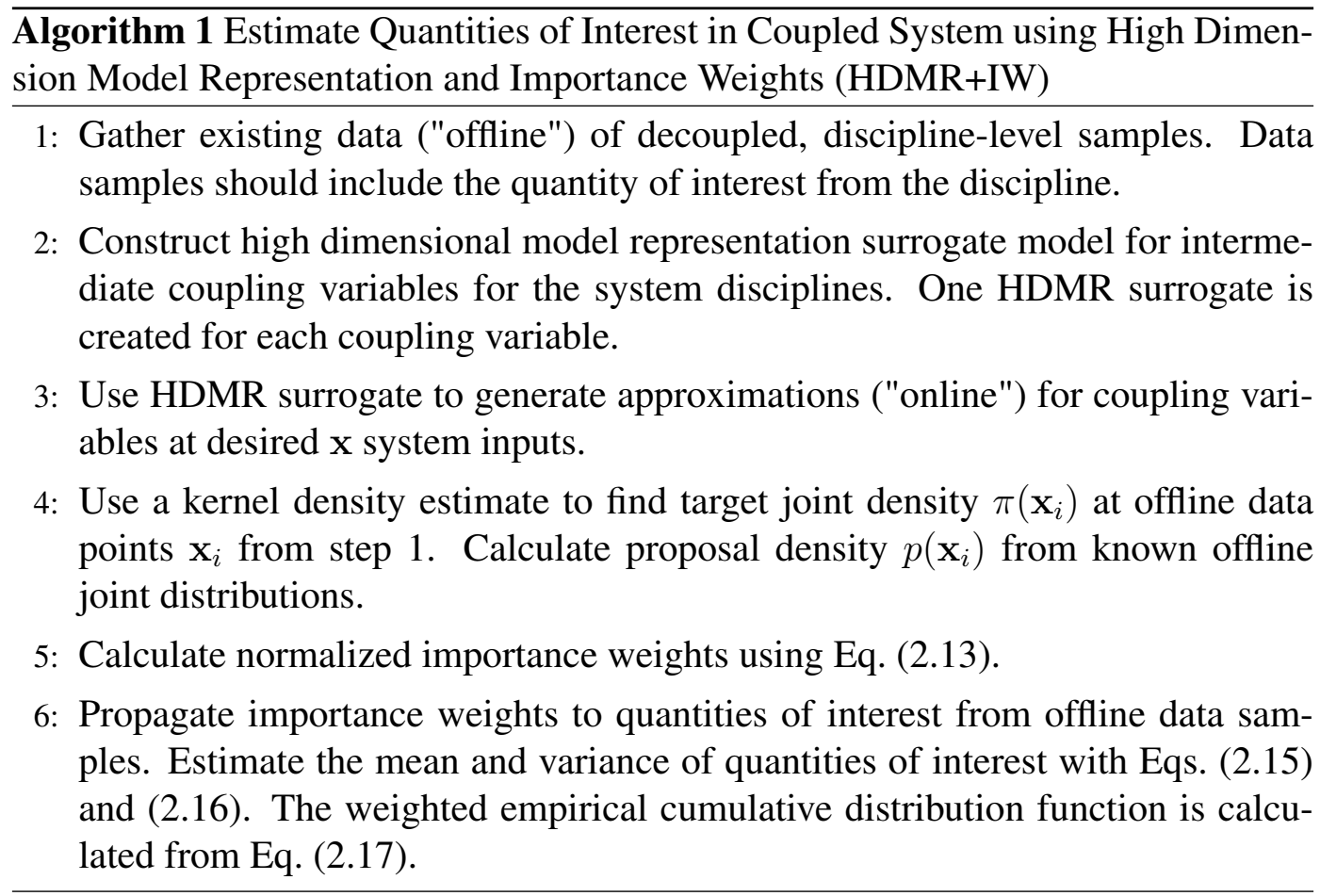

smoothed and then differentiated to get the respective probability density function.

\subsection{Algorithm}

The full step-by-step process of obtaining the dependent quantities of interest from the coupled system is described in Algorithm 1. The only full coupled system evaluations occur in Step 2 during the construction of the cut-HDMR surrogate model. The remaining computations only involve statistical analysis of the resulting data sets. If the coupled system in question is a highfidelity multiphysics model, then the combined statistical computations are computationally cheap compared to a full system evaluation. 


\section{RESULTS*}

\subsection{Results}

In this section we apply our cut high dimensional model representation plus importance weighting methodology $(\mathrm{HDMR}+\mathrm{IW})$ to estimate the quantities of interest from two coupled disciplines of a system. The estimates are validated from the results of Monte Carlo sampling. The method is implemented on an analytical example and a fire detection satellite system example.

\subsection{A Analytical Example}

The example presented here is based upon a three discipline analytical problem first described in Ref. [36]. Subsequently, Ref. [37] used a modified version of this model that only uses the two main disciplines. Our model uses the two disciplines with feedback coupling and each discipline will only have one stochastic input for each discipline. The remaining inputs in the model in Ref. [37] are set equal to one. The system used in the analysis is shown in Fig. 3.1 has $x_{1}, x_{2} \sim$ $\mathcal{N}(1,0.1)$ as stochastic inputs, $y_{12}$ and $y_{21}$ as coupling variables, and $q_{1}$ and $q_{2}$ as system quantities of interest. The offline distributions were chosen to be normal distributions with equal means but wider support than the distributions of the known system variables. The offline distributions were as follows: $x_{1, \text { off }} \sim \mathcal{N}(1,0.5)$ and $y_{21, \text { off }} \sim \mathcal{N}(11.9,1.225)$ as inputs for discipline 1 , and $x_{2, \text { off }} \sim \mathcal{N}(1,0.5)$ and $y_{12, \text { off }} \sim \mathcal{N}(8.9,1.225)$ as inputs for discipline 2.

Monte Carlo runs were used as a truth model to validate the results of our HDMR+IW methodology. One thousand samples were used for the Monte Carlo simulation, which required each discipline to be evaluated 14,999 times as part of fixed point iteration convergence. The cut-HDMR calculation used 8 samples on the cut vector in Eqs. (2.3), (2.4) and used up to $10^{\text {th }}$ degree Legendre polynomials for the basis functions in Eq. (2.5). The amount of times the cut-HDMR process does a full system evaluation is dependent upon the dimension of the system input and the number of samples used on the cut vector. For the analytical problem presented, the HDMR+IW method

\footnotetext{
${ }^{*}$ Reprinted from Ref. [2]
} 


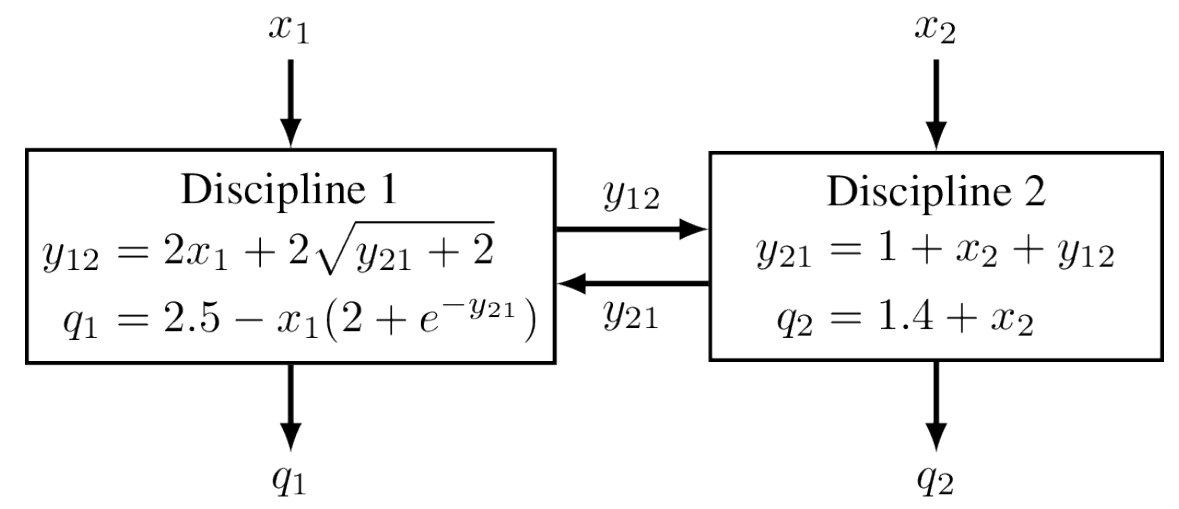

Figure 3.1: Analytical system example

Table 3.1: Results of analytical example

\begin{tabular}{ccccccccc}
\hline Method & $\operatorname{mean}\left(y_{12}\right)$ & $\operatorname{std}\left(y_{12}\right)$ & $\operatorname{mean}\left(y_{21}\right)$ & $\operatorname{std}\left(y_{21}\right)$ & $\operatorname{mean}\left(q_{1}\right)$ & $\operatorname{std}\left(q_{1}\right)$ & $\operatorname{mean}\left(q_{2}\right)$ & $\operatorname{std}\left(q_{2}\right)$ \\
\hline MC & $9.66 e+0$ & $2.79 e-1$ & $1.27 e+1$ & $3.10 e-1$ & $5.03 e-1$ & $2.01 e-1$ & $2.40 e+0$ & $9.64 e-2$ \\
HDMR+IW & $9.63 e+0$ & $3.04 e-1$ & $1.26 e+1$ & $3.34 e-1$ & $4.82 e-1$ & $2.04 e-1$ & $2.40 e+0$ & $1.03 e-1$ \\
Offline & $8.90 e+0$ & $1.22 e+0$ & $1.19 e+1$ & $1.23 e+0$ & $4.97 e-1$ & $1.00 e+0$ & $2.40 e+0$ & $4.99 e-1$ \\
\hline
\end{tabular}

required 81 full system samples which resulted in each discipline being evaluated 1,199 times.

The means and standard deviations for each coupling variable and quantity of interest in the analytical example are displayed in Table 3.1. The MC method represents the Monte Carlo evaluations of the system. The HDMR+IW method is Algorithm 1 described in Section 2.1. The offline method represents the statistics of the previously available offline samples for each discipline. The statistics for the offline quantities of interest were calculated from the result of the evaluation of the offline input samples in a decoupled state shown in Fig. 1.1b.

The results show that the HDMR+IW method was able to successfully shift the distribution of the existing offline samples to match that of the Monte Carlo "truth" method. The impact of the importance weights can be more easily observed in the empirical cumulative distribution functions presented in Fig. 3.2. The HDMR+IW method took advantage of the existing offline samples from decoupled discipline evaluations and, with only an additional 81 coupled system evaluations, 
weighted those offline samples to closely approximate the truth model.

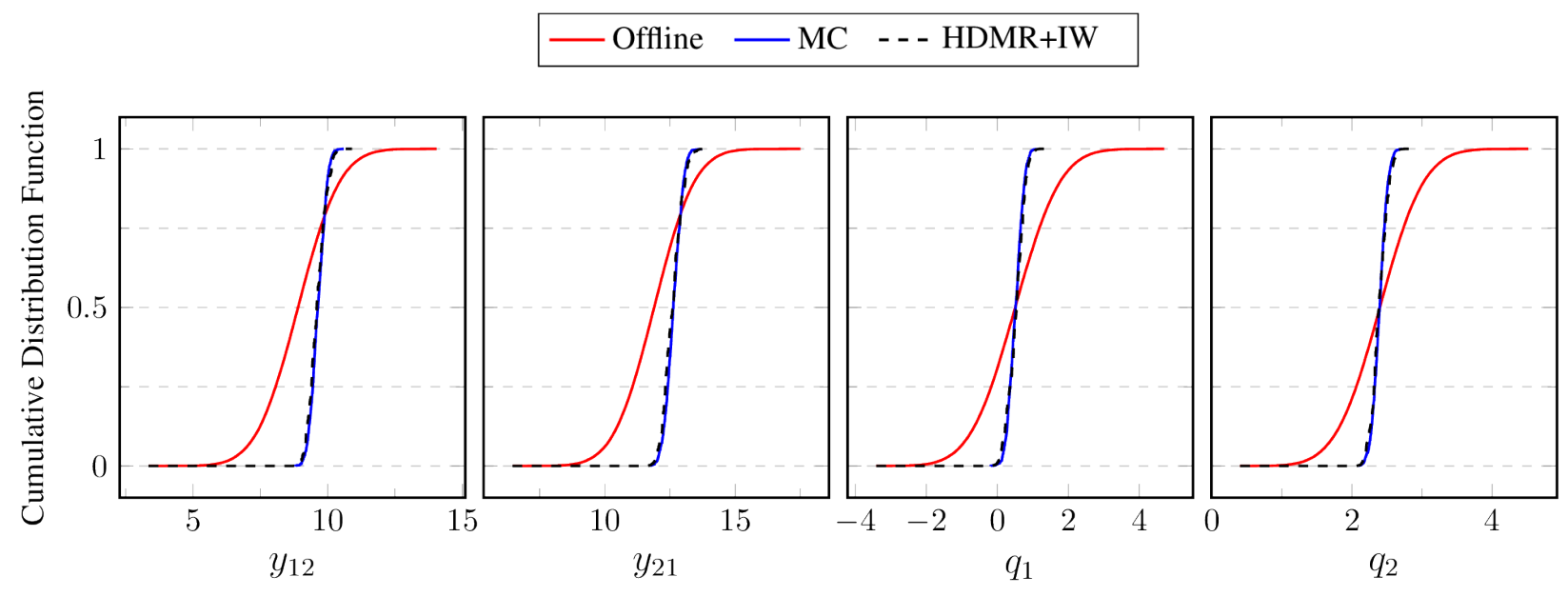

Figure 3.2: Cumulative distribution function of coupling variables $y_{12}$ and $y_{21}$, and quantities of interest $q_{1}$ and $q_{2}$ of analytical system example.

\subsection{B Fire Detection Satellite Example}

In the second demonstration, our HDMR+IW methodology is implemented on a coupled fire detection satellite model. The model is derived from Ref. [38] and reduces the original three discipline system down to the two coupled disciplines, which is shown in Fig. 3.3. The system has eight stochastic input variables with defined distributions displayed in Table 3.2. The system equations and constant parameters are listed in Ref. $[38,1]$. The system has three coupling variables: power of the attitude control system, $P_{\mathrm{ACS}}$, maximum moment of inertia, $I_{\max }$ and the minimum moment of inertia, $I_{\min }$. In a standard system evaluation, the values for these variables are found through a fixed point iteration scheme. There are three quantities of interest that are outcomes of the system. The quantity of interest for the attitude control discipline is the total torque, $\tau_{\text {tot. }}$ The power discipline has two quantities of interest: the total power output, $P_{\text {tot }}$ and the area of the solar array, $A_{\text {sa }}$. 
Average Solar Flux $\left(F_{s}\right)$

Deviation of Moment Axis from Vertical $(\theta)$

Solar Radiation Torque $\left(L_{s p}\right)$

Reflectance Factor $(q)$

Residual Dipole $\left(R_{D}\right)$

Aerodynamic Torque of Moment $\operatorname{Arm}\left(L_{a}\right)$

Drag Coefficient $\left(C_{d}\right)$

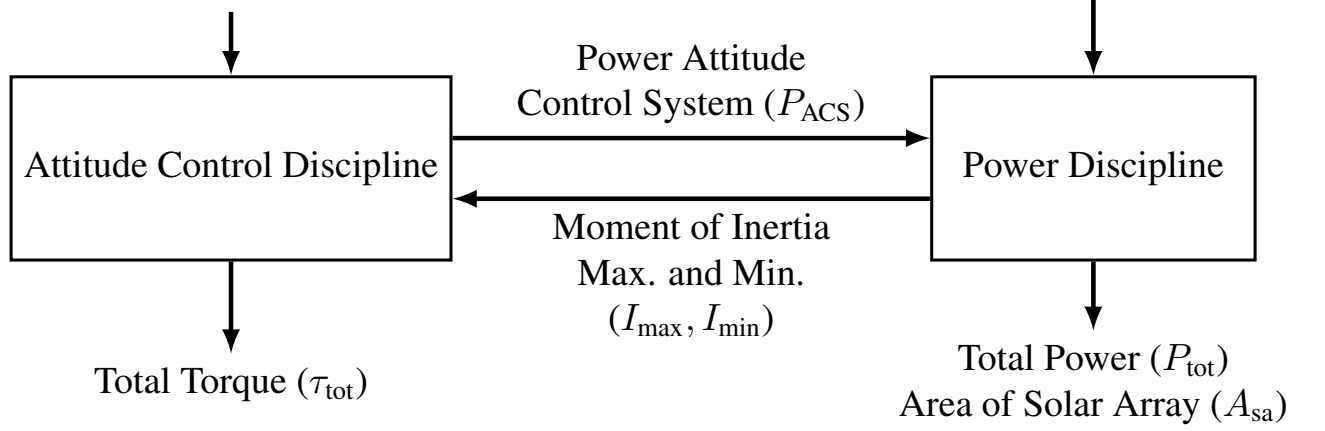

Figure 3.3: Fire detection satellite model

The offline input samples for the coupling variables were determined by first running a separate Monte Carlo simulation on the fire satellite system to view the empirical distributions under coupled conditions. Each coupling variable's offline distribution was then set to be Gaussian with the same mean as the empirical data and a standard deviation multiplied by a constant in order to increase the support for the offline samples. The multiplicative constant was set to 1.75 so that the offline distributions would have the necessary support to overlap the samples generated through the Monte Carlo simulation, which we take as the online "truth" model. The same 1.75 constant was used to increase the standard deviation of the system inputs to define their offline distributions. Table 3.2 lists the online and offline input distributions for the fire detection satellite example. One hundred thousand samples were drawn from the offline samples and sent once through the individual disciplines in the manner shown in Fig. $1.1 \mathrm{~b}$ to create 100,000 offline samples of the quantities of interest. 
Table 3.2: Fire detection satellite input variables as modified from Ref. [1]

\begin{tabular}{|c|c|c|c|c|}
\hline Random Variable & Symbol & Online & Offline & Units \\
\hline Power other than attitude control & $P_{\text {other }}$ & $\mathcal{N}(1000,50)$ & $\mathcal{N}(1000,87.5)$ & $W$ \\
\hline Average solar flux & $F_{s}$ & $\mathcal{N}(1400,20)$ & $\mathcal{N}(1400,35)$ & $W / m^{2}$ \\
\hline Deviation of moment axis & $\theta$ & $\mathcal{N}(15,1)$ & $\mathcal{N}(15,1.75)$ & deg \\
\hline Moment arm for radiation torque & $L_{s p}$ & $\mathcal{N}(2,0.4)$ & $\mathcal{N}(2,0.7)$ & $m$ \\
\hline Reflectance factor & $q$ & $\mathcal{N}(0.5,0.1)$ & $\mathcal{N}(0.5,0.175)$ & \\
\hline Residual dipole of spacecraft & $R_{D}$ & $\mathcal{N}(5,1)$ & $\mathcal{N}(5,1.75)$ & $\mathrm{A} \cdot \mathrm{m}^{2}$ \\
\hline Moment arm for aerodynamic torque & $L_{a}$ & $\mathcal{N}(2,0.4)$ & $\mathcal{N}(2,0.7)$ & $\mathrm{m}$ \\
\hline Drag coefficient & $C_{d}$ & $\mathcal{N}(1,0.3)$ & $\mathcal{N}(1,0.535)$ & \\
\hline Power attitude control system & $P_{\text {ACS }}$ & - & $\mathcal{N}(130,26.4)$ & $W$ \\
\hline Maximum moment of inertia & $I_{\max }$ & - & $\mathcal{N}(6613,31.9)$ & $\mathrm{kg} \cdot \mathrm{m}^{2}$ \\
\hline Minimum moment of inertia & $I_{\min }$ & - & $\mathcal{N}(5116,32.2)$ & $\mathrm{kg} \cdot \mathrm{m}^{2}$ \\
\hline
\end{tabular}

The increased number of input and coupling variables raises the dimensionality of the kernel density estimate from 4 in the analytical example to 11 in the fire detection satellite model. In this case, the approximation was still reasonable, however, systems with a higher number of input and coupling variables could be susceptible to convergence issues with high dimensional kernel density estimates [32, 39].

Since the fire satellite model takes 8 stochastic inputs to the coupled system, the cut-HDMR process would require significantly more function evaluations to generate the approximations. We reduced the cut-HDMR's expense by lowering the number of cut vector samples from 8 to 6 . Ref. [9] demonstrates empirically that the approximation error does not marginally improve with more than 6 samples in the cut vector. Further, most bivariate terms result in negligible improvement in the overall approximation and thus, can not be computed. This would result in substantial savings (possibly only 48 total evaluations) if this information were known a priori. A careful 
study of this is a topic of future work. Using the new cut samples with both univariate and bivariate terms, the HDMR+IW method required 1,009 system evaluations and 3,027 evaluations of each individual discipline. The Monte Carlo "truth" model required 10,000 system evaluations and 30,128 evaluations of each discipline.

The empirical cumulative distribution functions of the coupling variables produced by Algorithm 1 and the Monte Carlo simulation are shown in Fig. 3.4.

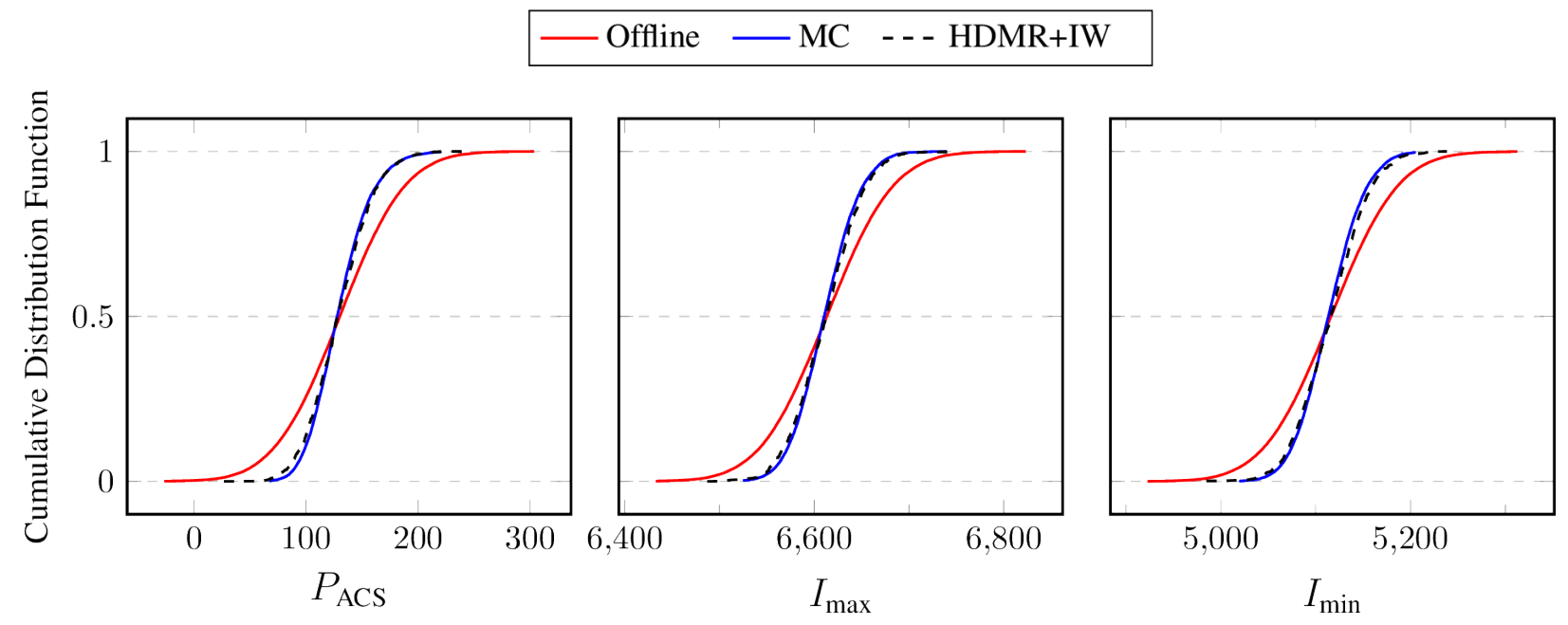

Figure 3.4: Fire Satellite: cumulative distribution functions of coupling variables: power other than attitude control, $P_{\mathrm{ACS}}$, maximum moment of inertia, $I_{\max }$ and minimum moment of inertia, $I_{\min }$.

Similarly, the results for the fire satellite model quantities of interest are shown in Fig. 3.5. 


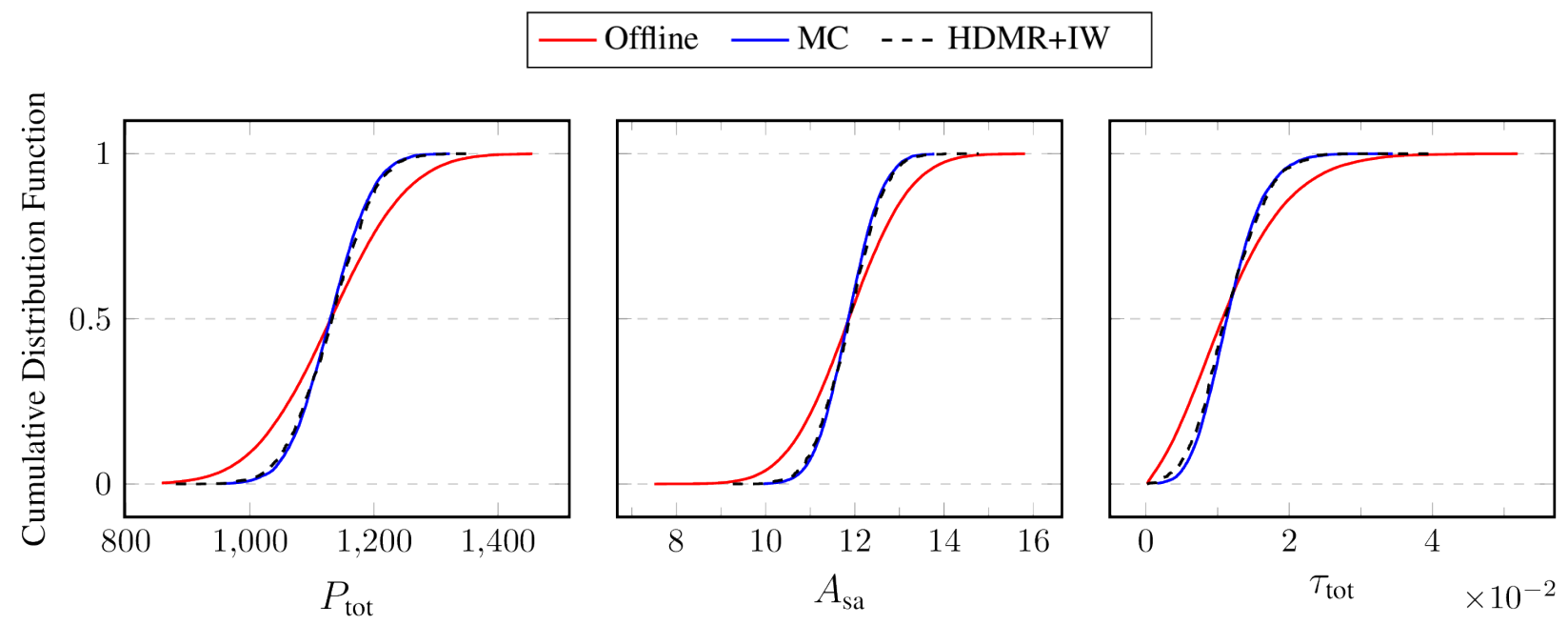

Figure 3.5: Fire Satellite: cumulative distribution functions of quantities of interest: total power, $P_{\text {tot }}$, area of solar array, $A_{\mathrm{sa}}$ and total torque $\tau_{\text {tot }}$.

The resulting mean and standard deviation of the coupling variables are displayed in Table 3.3, while the statistics for the quantities of interest are shown in Table 3.4.

Table 3.3: Results of fire satellite example, coupling variables

\begin{tabular}{ccccccc}
\hline Method & $\operatorname{mean}\left(P_{\mathrm{ACS}}\right)$ & $\operatorname{std}\left(P_{\mathrm{ACS}}\right)$ & $\operatorname{mean}\left(I_{\max }\right)$ & $\operatorname{std}\left(I_{\max }\right)$ & $\operatorname{mean}\left(I_{\min }\right)$ & $\operatorname{std}\left(I_{\min }\right)$ \\
\hline MC & $1.299 e+2$ & $2.552 e+1$ & $6.611 e+3$ & $3.151 e+1$ & $5.114 e+3$ & $3.182 e+1$ \\
HDMR+IW & $1.294 e+2$ & $2.738 e+1$ & $6.612 e+3$ & $3.462 e+1$ & $5.116 e+3$ & $3.535 e+1$ \\
Offline & $1.302 e+2$ & $4.617 e+1$ & $6.613 e+3$ & $5.580 e+1$ & $5.116 e+3$ & $5.612 e+1$ \\
\hline
\end{tabular}

Table 3.4: Results of fire satellite example, quantities of interest

\begin{tabular}{ccccccc}
\hline Method & $\operatorname{mean}\left(P_{\text {tot }}\right)$ & $\operatorname{std}\left(P_{\text {tot }}\right)$ & $\operatorname{mean}\left(A_{\mathrm{sa}}\right)$ & $\operatorname{std}\left(A_{\mathrm{sa}}\right)$ & $\operatorname{mean}\left(\tau_{\text {tot }}\right)$ & $\operatorname{std}\left(\tau_{\text {tot }}\right)$ \\
\hline MC & $1.130 e+3$ & $5.525 e+1$ & $1.186 e+1$ & $6.067 e-1$ & $1.166 e-2$ & $4.254 e-3$ \\
HDMR+IW & $1.131 e+3$ & $5.938 e+1$ & $1.187 e+1$ & $6.522 e-1$ & $1.147 e-2$ & $4.639 e-3$ \\
Offline & $1.130 e+3$ & $9.940 e+1$ & $1.187 e+1$ & $1.087 e+0$ & $1.177 e-2$ & $7.462 e-3$ \\
\hline
\end{tabular}


The HDMR+IW method provided a good approximation to the truth model with a full order of magnitude decrease in the required number of full system evaluations. The importance weights shifted the offline empirical distribution functions close to the Monte Carlo results, though with some noticeable error near the extremes of the distributions. The kernel density estimate is sufficient, though future work could explore using different bandwidth values or kernel functions. The importance weights similarly shifted the empirical distribution functions of the quantities of interest in Fig. 3.5. The total torque, $\tau_{\text {tot }}$, approximation has some error near left hand side of the distribution. The torque value is always positive, and most likely does not resemble a Gaussian distribution near zero, potentially introducing the error due to the use of the multivariate Gaussian kernel function in the density estimate. The discrepancy in the total torque approximation can also be seen in the sufficient statistics. The offline mean value of the total torque is slightly closer to the truth model than the approximation's mean value as seen in Table 3.4. Additional work is needed to quantify the error, which is necessary when determining the minimum tolerance of HDMR+IW approximation. The $\epsilon$ value in Eq. (2.10) can also be reduced to force a closer approximation if necessary.

The offline and online marginal joint densities of $A_{\mathrm{sa}}$ and $\tau_{\text {tot }}$ are shown in Fig. 3.6. The contours were plotted using a separate bivariate kernel density estimate with a Gaussian kernel function. A subset of the offline samples are shown in red on the left plot on top of the offline density contours in red. The online density contours are in blue. The right plot shows how the importance weighting step emphasizes the offline samples that exist at the center of the online density. The samples are plotted with their size proportional to the weight they were given. 

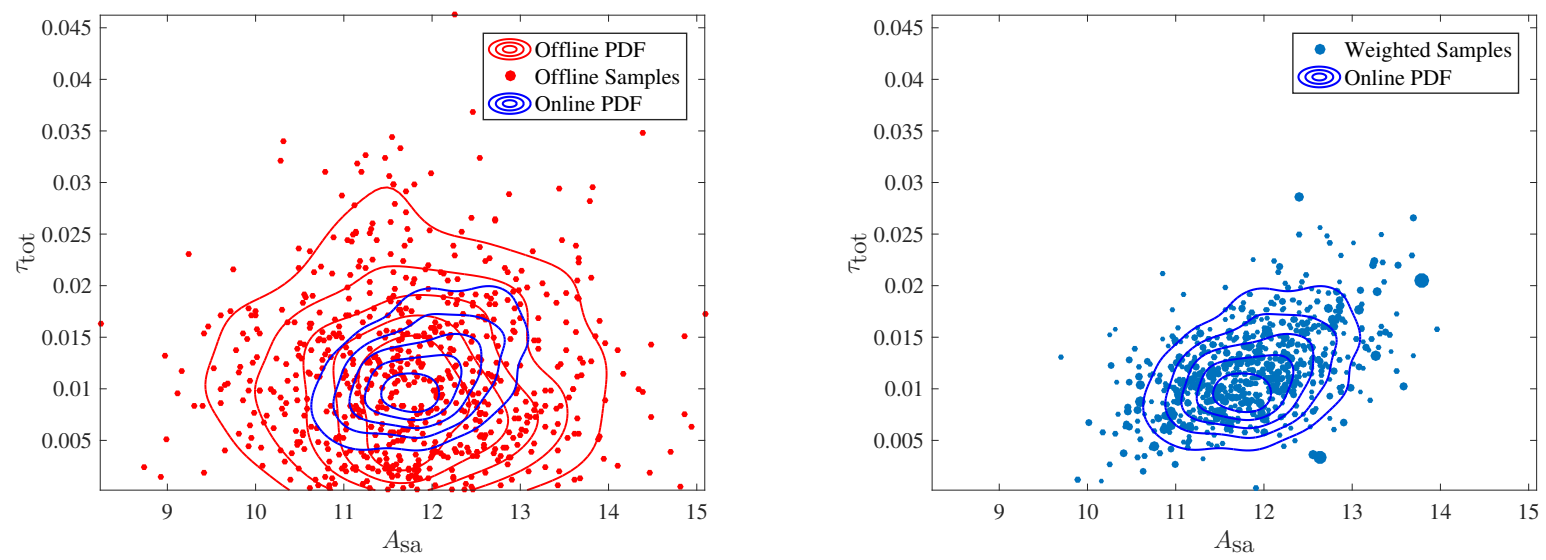

Figure 3.6: Illustration of importance weighting offline samples for the joint density of fire satellite model quantities of interest: area of the solar array, $A_{\mathrm{sa}}$, and total torque, $\tau_{\text {tot }}$. 


\section{FUTURE WORK}

\subsection{Future Work}

In this section we discuss the areas of opportunity for future work improving this approximation method. We review a multi-fidelity setup of the coupled system as well as an analysis of the system configuration.

\subsection{A Multi-fidelity Analysis}

This methodology for utilizing a library of decoupled offline samples lends itself to multifidelity analysis. We consider the prospect of redoing the system-level analysis after swapping in an equivalent higher fidelity discipline, seen in Fig. 4.1. If we wished to redo the analysis to obtain new values for the quantities of interest using the multi-fidelity full system model, we would still need to perform cut-HDMR, density estimation of the approximated coupling variables, and importance weighting the existing offline samples. This process assumes that a library exists of decoupled evaluations of the high-fidelity discipline, similar to our original setup. Note that full multi-fidelity system evaluations are still only performed at the cut-HDMR step, so we need only evaluate the high-fidelity discipline for the required number of online samples.

\subsection{B Network Topology Analysis}

The effectiveness of the HDMR+IW method of approximating the output quantities of interest is dependent upon the structure of the system. The location of the intermediate coupling variables and arrangement of disciplines with feedback coupling will determine the number of online full system evaluations required to build the cut-HDMR surrogate models. The HDMR+IW method utilizes an offline library of discipline-level sample distributions to compose an approximation for a system-level quantity of interest. It is possible that certain network topology arrangements are less favorable to HDMR+IW approximations than using direct surrogate models for systemlevel quantities of interest. A network analysis of generic multidisciplinary systems with varying amounts of feed-forward and feed-back coupling between disciplines could provide insight on 
Average Solar Flux $\left(F_{s}\right)$

Deviation of Moment Axis from Vertical $(\theta)$

Solar Radiation Torque $\left(L_{s p}\right)$

Reflectance Factor $(q)$

Residual Dipole $\left(R_{D}\right)$

Aerodynamic Torque of Moment $\operatorname{Arm}\left(L_{a}\right)$

Drag Coefficient $\left(C_{d}\right)$

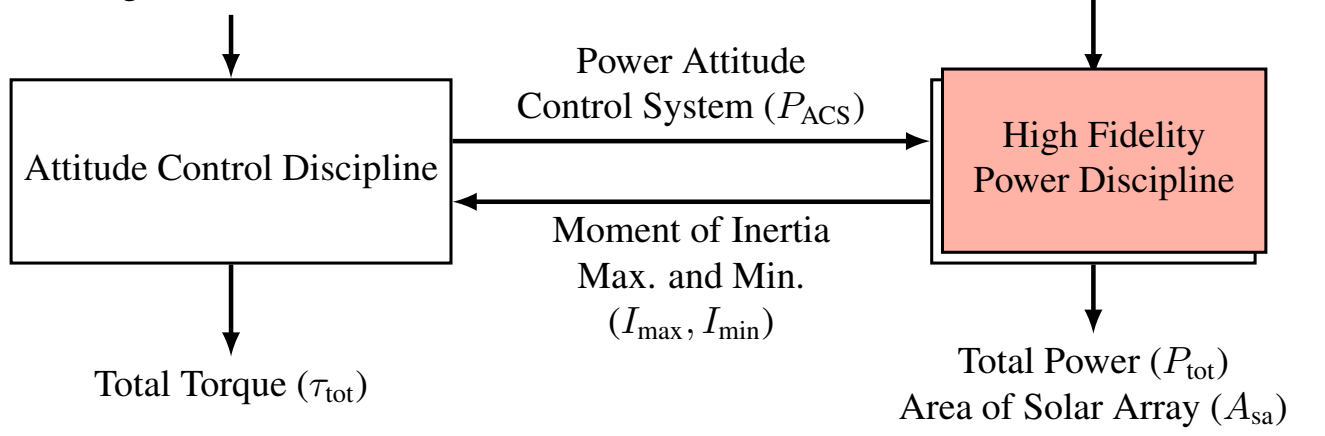

Figure 4.1: High-fidelity drop-in discipline replacement for fire satellite problem

whether a particular system's design is more favorable to HDMR+IW approximations as opposed to other surrogate methods. 


\section{CONCLUSIONS*}

\subsection{Conclusions}

We have demonstrated a method to approximate the statistics of quantities of interest derived from coupled multiphysics systems. The method takes advantage of existing discipline-level, decoupled samples through a procedure involving high dimensional model representation, kernel density estimates, and importance weighting the offline sample distributions. We were able to achieve a good approximation for the quantities of interest for a Monte Carlo simulation with an order of magnitude fewer full coupled system evaluations. This method is an inexpensive, effective way to propagate uncertainty in the inputs to a coupled system.

This work has also created several avenues for future research. In particular, a careful study of when bivariate terms may be ignored in the cut-HDMR approximations could result in even more substantial computational savings. A study of the impact of the error tolerance in the $\ell_{1}$ minimization could also lead to more savings, albeit with more approximation error, which may be deemed acceptable. This work has also created a rapid capability for seeing the results from modifying the discipline level input distributions. The online mapping would not need to be recomputed in this case and only new importance weights would be required. This creates the possibility for studying the sensitivity to input distributions without any further model evaluations. Lastly, this work has created the potential to quickly drop-in a new disciplinary model if it has been previously evaluated offline. All that is required is the online approximation of the new map from inputs to resolved coupling variables. This ability is quite practical, particularly in situations where certain decision makers prefer to use their models over other available options that have already been implemented in a given system.

\footnotetext{
${ }^{*}$ Reprinted from Ref. [2]
} 


\section{REFERENCES}

[1] S. Sankararaman and S. Mahadevan, "Likelihood-based approach to multidisciplinary analysis under uncertainty," Journal of Mechanical Design, vol. 134, no. 3, pp. 031008, 1-12, 2012.

[2] S. Friedman, B. Isaac, and D. L. Allaire, "Efficient Decoupling of Multiphysics Systems for Uncertainty Propagation," in 20th AIAA Non-Deterministic Approaches Conference, AIAA SciTech Forum, American Institute of Aeronautics and Astronautics, January 2018.

[3] M. C. Kennedy and A. O'Hagan, "Bayesian calibration of computer models," Journal of the Royal Statistical Society: Series B (Statistical Methodology), vol. 63, no. 3, pp. 425-464, 2001.

[4] S. Amaral, D. Allaire, and K. Willcox, "A decomposition approach to uncertainty analysis of multidisciplinary systems," in 12th AIAA Aviation Technology, Integration, and Operations (ATIO) Conference and 14th AIAA/ISSMO Multidisciplinary Analysis and Optimization Conference, 2012.

[5] S. Amaral, D. Allaire, and K. Willcox, "A decomposition-based approach to uncertainty analysis of feed-forward multicomponent systems," International Journal for Numerical Methods in Engineering, vol. 100, no. 13, pp. 982-1005, 2014.

[6] S. Ghoreishi and D. Allaire, "Compositional Uncertainty Analysis via Importance Weighted Gibbs Sampling for Coupled Multidisciplinary Systems," 18th AIAA Non-Deterministic Approaches Conference, no. January, pp. 1-15, 2016.

[7] S. Ghoreishi and D. Allaire, "Adaptive uncertainty propagation for coupled multidisciplinary systems," AIAA Journal, pp. 1-11, 2017. 
[8] A. Chaudhuri and K. Willcox, "Multifidelity uncertainty propagation in coupled multidisciplinary systems," in 18th AIAA Non-Deterministic Approaches Conference, p. 1442, 2016.

[9] B. Isaac, S. Friedman, and D. Allaire, "Efficient approximation of coupling variable fixed point sets for decoupling multidisciplinary systems," in AIAA/ASCE/AHS/ASC Structures, Structural Dynamics, and Materials Conference, 2018.

[10] J. Martin and T. Simpson, "A methodology to manage system-level uncertainty during conceptual design,” Journal of Mechanical Design, vol. 128, no. 4, pp. 959-968, 2006.

[11] W. Yao, X. Chen, W. Luo, M. van Tooren, and J. Guo, "Review of uncertainty-based multidisciplinary design optimization methods for aerospace vehicles," Progress in Aerospace Sciences, vol. 47, no. 6, pp. 450-479, 2011.

[12] X. S. Gu, J. Renaud, and C. Penninger, "Implicit uncertainty propagation for robust collaborative optimization," Journal of Mechanical Design, vol. 128, no. 4, pp. 1001-1013, 2006.

[13] A. Chiralaksanakul and S. Mahadevan, "Decoupled approach to multidisciplinary design optimization under uncertainty," Optimization and Engineering, vol. 8, no. 1, pp. 21-42, 2007.

[14] M. McDonald, K. Zaman, and S. Mahadevan, "Uncertainty quantification and propagation for multidisciplinary system analysis," in 12th AIAA/ISSMO Multidisciplinary Analysis and Optimization Conference, Paper 2008-6038, 2008.

[15] X. Du and W. Chen, "An efficient approach to probabilistic uncertainty analysis in simulationbased multidisciplinary design," in Proceedings of the 8th AIAA Symposium on Multidisciplinary Analysis and Optimization, 2000.

[16] Z. Jiang, W. Li, D. W. Apley, and W. Chen, "A system uncertainty propagation approach with model uncertainty quantification in multidisciplinary design," in ASME 2014 International Design Engineering Technical Conferences and Computers and Information in Engineering Conference, American Society of Mechanical Engineers, 2014. 
[17] M. Kokkolaras, Z. P. Mourelatos, and P. Y. Papalambros, "Design optimization of hierarchically decomposed multilevel systems under uncertainty," Journal of Mechanical Design, vol. 128 , no. 2 , pp. 503-508, 2006.

[18] X. Du and W. Chen, "Collaborative reliability analysis under the framework of multidisciplinary systems design," Optimization and Engineering, vol. 6, no. 1, pp. 63-84, 2005.

[19] S. Mahadevan and N. Smith, "Efficient first-order reliability analysis of multidisciplinary systems," International Journal of Reliability and Safety, vol. 1, no. 1, pp. 137-154, 2006.

[20] M. Arnst, R. Ghanem, E. Phipps, and J. Red-Horse, "Dimension reduction in stochastic modeling of coupled problems," International Journal for Numerical Methods in Engineering, vol. 92, no. 11, pp. 940-968, 2012.

[21] M. Arnst, R. Ghanem, E. Phipps, and J. Red-Horse, "Measure transformation and efficient quadrature in reduced-dimensional stochastic modeling of coupled problems," International Journal for Numerical Methods in Engineering, vol. 92, no. 12, pp. 1044-1080, 2012.

[22] M. Arnst, R. Ghanem, E. Phipps, and J. Red-Horse, "Reduced chaos expansions with random coefficientsin reduced-dimensional stochastic modeling of coupled problems," International Journal for Numerical Methods in Engineering, vol. 97, no. 5, pp. 352-376, 2014.

[23] P. G. Constantine, E. T. Phipps, and T. M. Wildey, "Efficient uncertainty propagation for network multiphysics systems," International Journal for Numerical Methods in Engineering, vol. 99, no. 3, pp. 183-202, 2014.

[24] M. Arnst, S. Craig, and R. Ghanem, "Hybrid sampling/spectral method for solving stochastic coupled problems," SIAM/ASA Journal on Uncertainty Quantification, vol. 1, no. 1, pp. 218243, 2013. 
[25] X. Chen, B. Ng, Y. Sun, and C. Tong, "A flexible uncertainty quantification method for linearly coupled multi-physics systems," Journal of Computational Physics, vol. 248, no. 1, pp. 383-401, 2013.

[26] H. Rabitz and Ö. F. Aliş, "General foundations of high-dimensional model representations," Journal of Mathematical Chemistry, vol. 25, no. 2-3, pp. 197-233, 1999.

[27] G. Li, C. Rosenthal, and H. Rabitz, "High dimensional model representations," The Journal of Physical Chemistry A, vol. 105, no. 33, pp. 7765-7777, 2001.

[28] I. Sobol', “Theorems and examples on high dimensional model representation,” Reliability Engineering and System Safety, vol. 79, pp. 187-193, 2003.

[29] M. Griebel and M. Holtz, "Dimension-wise integration of high-dimensional functions with applications to finance," Journal of Complexity, vol. 26, no. 5, pp. 455-489, 2010.

[30] K. Li and D. Allaire, "A compressed sensing approach to uncertainty propagation for approximately additive functions," in ASME 2016 International Design Engineering Technical Conferences and Computers and Information in Engineering Conference, pp. V01AT02A027V01AT02A027, American Society of Mechanical Engineers, 2016.

[31] E. J. Candès, J. Romberg, and T. Tao, "Robust uncertainty principles: Exact signal reconstruction from highly incomplete frequency information," IEEE Transactions on information theory, vol. 52, no. 2, pp. 489-509, 2006.

[32] D. W. Scott, Multivariate Density Estimation: Theory, Practice, and Visualization. Somerset, United States: Wiley, 2015.

[33] S. Amaral, D. Allaire, and K. Willcox, "A Decomposition-Based Approach to Uncertainty Analysis of Feed-Forward Multicomponent Systems," International Journal for Numerical Methods in Engineering, vol. 100, pp. 982-1005, dec 2014. 
[34] J. S. Liu, "Metropolized independent sampling with comparisons to rejection sampling and importance sampling," Statistics and Computing, vol. 6, no. 2, pp. 113-119, 1996.

[35] A. F. M. Smith and A. E. Gelfand, "Bayesian Statistics without Tears : A SamplingResampling Perspective,” The American Statistician, vol. 46, no. 2, pp. 84-88, 1992.

[36] X. Du and W. Chen, "Collaborative reliability analysis under the framework of multidisciplinary systems design," Optimization and Engineering, vol. 6, no. 1, pp. 63-84, 2005.

[37] S. Mahadevan, Sankaran Natasha, "Efficient first-order reliability analysis of multidisciplinary systems," International Journal of Reliability and Safety, vol. 1, no. 1/2, pp. 137-154, 2006.

[38] K. Zaman and S. Mahadevan, "Reliability-based design optimization of multidisciplinary system under aleatory and epistemic uncertainty," Structural and Multidisciplinary Optimization, vol. 55, no. 2, pp. 681-699, 2017.

[39] T. Nagler and C. Czado, "Evading the curse of dimensionality in nonparametric density estimation with simplified vine copulas," Journal of Multivariate Analysis, vol. 151, pp. 69-89, 2016. 